\title{
Modern Techniques in Galaxy Kinematics: CDI and the Planetary Nebula Spectrograph
}

N. G. Douglas, K. Kuijken, A.J. Romanowsky, M.R. Merrifield, M. Arnaboldi, K. Freeman, K. Taylor

Members of the PN.Spectrograph Team

\begin{abstract}
We report here the successful commissioning of the PN.Spectrograph, the first special-purpose instrument for the measurement of galaxy kinematics through the $\mathrm{PN}$ population.
\end{abstract}

\section{Introduction}

Planetary nebulae: to most of the participants at this conference, beautiful objects displaying a range of structures, spherical, bipolar and rhomboidal, riotous colours, and the complex spectral signature of ionised gases, shocks, and dust. To others, the extragalactic crowd, they are seen as featureless points of light with the simplest of all possible spectra, a solitary green emission line.

\section{Observations of $\mathrm{PNe}$ in external galaxies}

The peculiar obervational properties of $\mathrm{PNe}$ at large distance have made them popular probes of galaxy dynamics. A large fraction of the total luminosity of the central star is re-emitted at the $5007 \AA$ [OIII] wavelength, making $\mathrm{PNe}$ easy to detect as individual objects even at the distances required by curent galaxy studies ( $\sim 20-30 \mathrm{Mpc})$. Detection is usually limited by the galaxy's own diffuse light, so PNe are most useful in the fainter, outer parts. Therefore they complement the use of integrated stellar spectroscopy, which is generally limited to the central 1-2 effective radii. Determining the radial velocities of the $\mathrm{PNe}$ is in principle straightforward on account of their narrow emission-line spectra.

At IAU Symposium 180 we reported plans for a new instrument, designed to measure the positions and radial velocities of $\mathrm{PNe}$ in a single observation. We are pleased to report that this "Planetary Nebula Spectrograph" was commissioned at the William Herschel Telescope (the $4.2 \mathrm{~m}$ telescope at La Palma Observatory) on July 16 2001, just a few months before the current meeting.

\section{Why a dedicated instrument?}

Detection of PNe in external galaxies is normally done by means of an onband/off-band survey to detect emission-line objects at the [OIII] wavelength, followed by multi-object spectroscopy to measure the radial velocities. These two steps are usually completely disjoint in terms of the instrument and telescope 

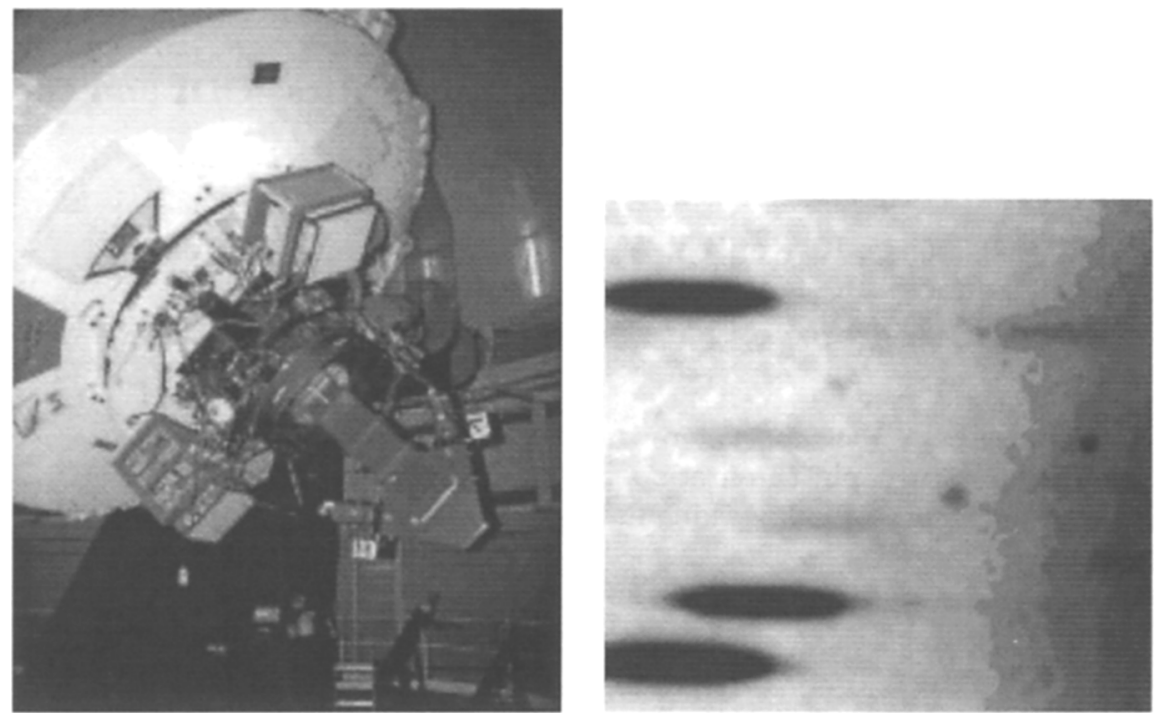

Figure 1. Left: the PN.S at the Cassegrain focus of the WHT - visible in the picture are the two cryostats whose axes point to the dual grating assembly at which the pupil is split between the two cameras. Right: a small section of a typical image, showing several PNe (points) as well as three bright and several fainter stars (extended)

used, leading to significant astrometric errors so that in practice the spectroscopy often returns unsatisfactory results in terms of completeness and accuracy.

The PN.Spectrograph uses slitless spectroscopy both to find the PNe and to measure their radial velocities. By splitting the light from the telescope between two gratings, a pair of images is obtained in which the dispersion directions are opposite: we call this counter-dispersed imaging (CDI). From these two frames, the velocities of the point-like objects can be obtained.

Thus, the entire observation consists of a single deep integration with the PN.Spectrograph. The total time spent is longer than that required by the traditional on-band/off-band survey, but not by much. For one thing, switching between filters is no longer required, since emission-line sources can be distinguished on the basis of their shape (see Fig. 1). Also, the dedicated design has allowed us to optimise all the components at $5007 \AA$ and thus reach high optical efficiency (33\%). More importantly, the entire multi-object spectroscopy stage is rendered redundant. The fact that (relative) velocities are determined by the displacement of point-like objects on the stable medium of a CCD lends itself to very high accuracy. The absolute velocity scale is fixed by use of a calibration mask which, like the field of interest, is registered simultaneously in both arms of the spectrograph. The PN.Spectrograph is currently returning exciting data and progress may be monitored through our website ${ }^{17}$.

${ }^{17}$ PN.S website: http://www.astro.rug.nl/ pns 